\title{
A RELAXATION OF STEINBERG'S CONJECTURE
}

\author{
OWEN HILL AND GEXIN YU
}

\begin{abstract}
A graph is $\left(c_{1}, c_{2}, \cdots, c_{k}\right)$-colorable if the vertex set can be partitioned into $k$ sets $V_{1}, V_{2}, \ldots, V_{k}$, such that for every $i: 1 \leq i \leq k$ the subgraph $G\left[V_{i}\right]$ has maximum degree at most $c_{i}$. We show that every planar graph without 4 - and 5 -cycles is $(1,1,0)$-colorable and $(3,0,0)$-colorable. This is a relaxation of the Steinberg Conjecture that every planar graph without 4 - and 5 -cycles are properly 3 -colorable (i.e., $(0,0,0)$-colorable).
\end{abstract}

\section{INTRODUCTION}

It is well-known that the problem of deciding whether a planar graph is properly 3-colorable is NP-complete. Grötzsch in 1959 [5] showed the famous theorem that every triangle-free planar graph is 3-colorable. A lot of research was devoted to find sufficient conditions for a planar graph to be 3-colorable, by allowing a triangle together with some other conditions. One of such efforts is the following famous conjecture made by Steinberg in 1976 .

Conjecture 1 (Steinberg, [7]). All planar graphs without 4-cycles and 5-cycles are 3-colorable.

Not much progress in this direction was made until Erdös proposed to find a constant $C$ such that a planar graph without cycles of length from 4 to $C$ is 3-colorable. Borodin, Glebov, Raspaud, and Salavatipour [2] showed that $C \leq 7$. For more results, see the recent nice survey by Borodin [1.

Yet another direction of relaxation of the Conjecture is to allow some defects in the color classes. A graph is $\left(c_{1}, c_{2}, \cdots, c_{k}\right)$-colorable if the vertex set can be partitioned into $k$ sets $V_{1}, V_{2}, \ldots, V_{k}$, such that for every $i: 1 \leq i \leq k$ the subgraph $G\left[V_{i}\right]$ has maximum degree at most $c_{i}$. Thus a $(0,0,0)$-colorable graph is properly 3 -colorable.

Eaton and Hull [4] and independently Škrekovski [6] showed that every planar graph is $(2,2,2)$-colorable (actually choosable). $\mathrm{Xu}$ [8] proved that all planar graphs with no adjacent triangles or 5-cycles are $(1,1,1)$-colorable. Chang, Havet, Montassier, and Raspaud [3] proved that all planar graphs without 4-cycles or 5-cycles are (2,1,0)-colorable and $(4,0,0)$-colorable. In this paper, we further prove the following relaxation of the Steinberg Conjecture.

Theorem 1. All planar graphs without 4-cycles and 5-cycles are (1,1,0)-colorable.

Theorem 2. All planar graphs without 4-cycles and 5-cycles are (3,0,0)-colorable.

We will use a discharging argument in the proofs. We let the initial charge of vertex $u \in G$ be $\mu(u)=2 d(u)-6$, and the initial charge of face $f$ be $\mu(f)=d(f)-6$. Then by Euler's formula, we have

$$
\sum_{v \in V(G)} \mu(u)+\sum_{f \in F(G)} \mu(f)=-12 .
$$

Date: November 1, 2018. 
Our goal is to show that we may re-distribute the charges among vertices and faces so the final charges of the vertices and faces are non-negative, which would be a contradiction. In the process of discharging, we will see that some configurations prevent us from showing some vertices or faces to have non-negative charges. Those configurations will be shown to be reducible configurations, that is, a valid coloring outside of the configurations can be extended to the whole graph. It is worth to note that in the proof of Theorem 1, we prove a somewhat global structure, a special chain of triangles, to be reducible.

The following are some simple observations about the minimal counterexamples to the above theorems.

Proposition 1. Among all planar graphs without 4-cycles and 5-cycles that are not $(1,1,0)$ colorable or $(3,0,0)$-colorable, let $G$ be one with minimum number of vertices. Then

(a) $G$ contains no $2^{-}$vertices.

(b) a $k$-vertex in $G$ can have $\alpha \leq\left\lfloor\frac{k}{2}\right\rfloor$ incident 3 -faces, and at most $k-2 \alpha$ pendant 3-faces.

We will use the following notations in the proofs. A $k$-vertex $\left(k^{+}\right.$-vertex, $k^{-}$-vertex $)$is a vertex of degree $k$ (at least $k$, at most $k$ resp.). The same notation will apply to faces. An $\left(\ell_{1}, \ell_{2}, \ldots, \ell_{k}\right)$-face is a $k$-face with incident vertices of degree $\ell_{1}, \ell_{2}, \ldots, \ell_{k}$. A bad 3-vertex is a 3 -vertex on a 3-face. A face $f$ is a pendant 3-face to vertex $v$ if $v$ is adjacent to some bad 3 -vertex on $f$. The pendant neighbor of a 3-vertex $v$ on a 3 -face is the neighbor of $v$ not on the 3-face. A vertex $v$ is properly colored if all neighbors of $v$ have different colors from $v$. A vertex $v$ is nicely colored if it shares colors with at $\operatorname{most} \max \left\{s_{i}-1,0\right\}$ neighbors, thus if a vertex $v$ is nicely colored by a color $c$ which allows deficiency $s_{i}>0$, then an uncolored neighbor of $v$ can be colored by $c$.

In the next section, we will give a proof to Theorem 1; and in the last section, we will give a proof to Theorem 2 .

\section{2. (1, 1,0)-COLORING OF PLANAR GRAPHS}

We will use a discharging argument in our proof. First we will prove some reducible configurations.

Let $G$ be a minimum counterexample to Theorem 1 , that is, $G$ is a planar graph without 4-cycles and 5-cycles, and $G$ is not $(1,1,0)$-colorable, but any proper subgraph of $G$ is $(1,1,0)$-colorable.

The following is a very useful tool in the proofs.

Lemma 1. Let $H$ be a proper subgraph of $G$ so that there is a $(1,1,0)$-coloring of $G-H$. If vertex $v \in H$ satisfies either (i) 3 neighbors of $v$ are colored, with at least two properly colored, or (ii) 4 neighbors of $v$ are colored, all properly, then the coloring of $G-H$ can be extended to $G-(H-v)$.

Proof. (i) Let $v \in H$ be a vertex with 3 colored neighbors, two of which are properly colored, such that the coloring of $G-H$ can not be extended to $v$. Since $v$ is not $(1,1,0)$-colorable, the three neighbors of $v$ must have different colors, and furthermore, two of the colored neighbors cannot be properly colored, a contradiction to the assumption that two of the colored neighbors of $v$ are properly colored.

(ii) Let $v \in H$ be a vertex of degree 4 with all neighbors properly colored such that the coloring of $G-H$ can not be extended to $v$. Then due to the coloring deficiencies, $v$ must have at least 2 neighbors colored by 1 , at least 2 neighbors colored by 2 , and at least 1 neighbor colored by 1 . Then $v$ has at least five colored neighbors, a contradiction. 
Lemma 2. There is no $\left(3,3,4^{-}\right)$-face in $G$.

Proof. Let $u v w$ be a $\left(3,3,4^{-}\right)$-face in $G$ with $d(u)=d(v)=3$ and $d(w) \leq 4$. Then $G \backslash\{u, v, w\}$ is $(1,1,0)$-colorable. Color $w$ and $v$ properly, then $u$ is colorable by Lemma 1 . thus $G$ is $(1,1,0)$-colorable, a contradiction.

Lemma 3. There is no 5-vertex that is incident to two $\left(3,4^{-}, 5\right)$-faces and adjacent to a 3-vertex in $G$.

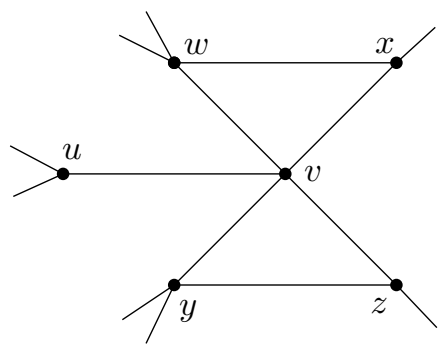

FiguRE 1. Figure for Lemma 3

Proof. Let $v$ be a 5-vertex with neighbors $u, w, x, y, z$ so that $w x, y z \in E(G)$ and $d(u)=$ $d(x)=d(z)=3$ and $d(w), d(y) \leq 4$ (See Figure 1). By the minimality of $G, G \backslash\{u, v, w, x, y, z\}$ is $(1,1,0)$-colorable. Properly color $u, w$, and $y$, then properly color $x$ and $z$. For $v$ to not be colorable, $v$ must have two neighbors colored by 1 , two neighbors colored by 2 and one neighbor colored by 3 . Since the $w, x$ and $y, z$ vertex pairs must be colored differently, one of them must have the colors 1 and 2. W.l.o.g. we can assume that $w$ is colored by 1 and $x$ by 2 . Then since $w$ is properly colored, we can either recolor $x$ by 1 or 3 , and color $v$ by 2 obtaining a coloring of $G$, a contradiction.

Lemma 4. No 3-vertex in $G$ can be adjacent to two other 3-vertices. In particular, the 3 -vertices on a $\left(3,3,5^{+}\right)$-face must have another neighbor with degree four or higher.

Proof. Let $v$ be a 3-vertex with $x$ and $y$ being two neighbors of degree 3. By the minimality of $G, G \backslash\{v, x, y\}$ is $(1,1,0)$-colorable. Then we can first properly color $x$ and $y$, and then by Lemma 1 color $v$ to get a coloring of $G$, a contradiction.

Lemma 5. The pendant neighbor of the 3-vertex on a $(3,4,4)$-face must have degree 4 or higher.
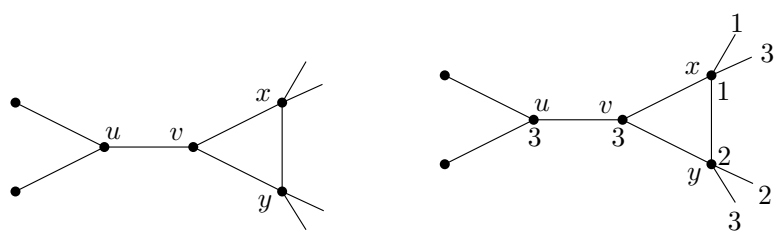

Figure 2. Figure for Lemma 5 
Proof. Let $v x y$ be a $(3,4,4)$-face in $G$ such that the pendant neighbor $u$ of the 3-vertex $v$ has degree 3 (See Figure 2). By the minimality of $G, G \backslash\{u, v\}$ is $(1,1,0)$-colorable. We properly color $u$ and then color $v$ differently from both $x$ and $y$. If $u$ and $v$ are not both colored by 3 , then we get a coloring for $G$, a contradiction, so we may assume both $u$ and $v$ are colored by 3 . This means that both $u$ and $v$ have two remaining neighbors colored by 1 and 2 . Let $x$ and $y$ be colored by 1 and 2 respectively. The neighbors of $x$ must be colored by 1 and 3 or else we could recolor $v$ by 1 and $x$ by 3 if necessary to obtain a coloring of $G$. Likewise, the neighbors of $y$ must be colored by 2 and 3. In this case we switch the colors of $x$ and $y$ and color $v$ by 1 to obtain a coloring of $G$, a contradiction again.

Let a $\left(T_{0}, T_{1}, \ldots, T_{n}\right)$-chain be a sequence of triangles, $T_{0}, T_{1}, \ldots, T_{n}$, such that (i) $T_{0}$ is a $(3,4,4)$-face and $T_{n}$ is a $\left(3^{+}, 4,4^{+}\right)$-face, and all other triangles are $(4,4,4)$-faces, and (ii) for $0 \leq i \leq n-1, T_{i}$ and $T_{i+1}$ share a 4 -vertex $t_{i}$. In a $\left(T_{0}, T_{1}, \ldots, T_{n}\right)$-chain, let $x_{i} \in T_{i}$ for $0 \leq i \leq n$ be a non-connecting $4^{+}$-vertex.

Let a special 4-vertex be a 4 -vertex that is incident to one 3 -face and has two pendant 3-faces, and let a 3-face be a special 3-face if it has at least one special 4-vertex. Let a good 4-vertex be a 4-vertex with only one incident 3 -face and at most one pendant 3 -face.

We will prove in the following lemmas that a $(3,4,4)$-face $T_{0}$ may get help in discharging from a $\left(3^{+}, 4^{+}, 5^{+}\right)$-face or special 3 -face $T_{n}$ through a $\left(T_{0}, T_{1}, \ldots, T_{n}\right)$-chain.

Lemma 6. There are no special $(3,4,4)$-faces in $G$.

Proof. Let $u v w$ be a special $(3,4,4)$-face in $G$ such that $d(v)=d(w)=4$. W.l.o.g. we can assume that $v$ is a special 4 -vertex with pendant neighbors $v_{1}$ and $v_{2}$. By the minimality of $G, G \backslash\left\{u, v, v_{1}, v_{2}, w\right\}$ is $(1,1,0)$-colorable. We can properly color $w$ and $u$ in that order then properly color $v_{1}$ and $v_{2}$. Then by Lemma 1, we can color $u$, obtaining a coloring of $G$, a contradiction.

The following is a very useful tool in extending a coloring to a chain.

Lemma 7. Consider a $\left(T_{0}, T_{1}, \cdots, T_{n}\right)$-chain with $n \geq 1$ and $T_{n}$ being $a\left(4,4^{-}, k\right)$-face. If $G \backslash\left\{T_{0}, T_{1}, \cdots, T_{n-1}\right\}$ has a coloring such that the $k$-vertex of $T_{n}$ is properly colored, or it shares the same color with the $4^{-}$-vertex, then the coloring can be extended to $G$.

Proof. We assume that the $\left(4,4^{-}, k\right)$-face $T_{n}$ has $k$-vertex $x_{n}$ and $4^{-}$-vertex $t_{n}$. Also let $G \backslash\left\{T_{0}, T_{1}, \cdots, T_{i-1}\right\}$ has a coloring such that $x_{n}$ is properly colored or shares the same color with $t_{n}$ and $G$ does not have a $(1,1,0)$-coloring. Finally let $u$ be the 3 -vertex of $T_{0}$ and let $w$ be the pendant neighbor of $u$.

We consider two cases. First let $n=1$. If $x_{1}$ and $t_{1}$ have the same color, then we can properly color $x_{0}$ and $t_{0}$ in that order, thus by Lemma 1 we can color $u$ so $G$ has a $(1,1,0)$ coloring, a contradiction. So we know that $x_{1}$ and $t_{1}$ must be colored differently, and further $x_{1}$ is colored properly. We can properly color $x_{0}$. If $x_{0}$ and $w$ share the same color then we can color $t_{0}$ by Lemma 1 and properly color $u$, a contradiction. So we may assume that $x_{0}$ and $w$ are colored differently. If any two of $x_{0}, x_{1}$, and $t_{1}$ are colored the same then we could color $t_{0}$ properly and color $u$ by Lemma 1, a contradiction. Since $x_{0}, x_{1}$, and $t_{1}$ are colored differently, if $x_{0}$ is not colored by 3 then we could color $t_{0}$ by the same color as $x_{0}$ and properly color $u$, a contradiction. So $x_{0}$ must be colored by 3 and w.l.o.g. we can assume that $w$ is colored by 1 . Since $x_{1}$ is properly colored, it must be colored by 2 , or we could color $t_{0}$ by 1 and properly color $u$, a contradiction. It follows that $t_{1}$ is colored by 1 . If $t_{1}$ is colored properly, then we could color $t_{0}$ by 1 and properly color $u$, a contradiction, so we may 
assume that $t_{1}$ is not colored properly. Further, neither $z$ nor $z^{\prime}$ (the two other neighbors of $t_{1}$ ) can be colored by 2 , or we could recolor $t_{1}$ properly, then color $t_{0}$ by 1 and $u$ properly, a contradiction. So we color $t_{1}$ by 2 and $t_{0}$ by 1 , and properly color $u$, a contradiction.

Now we assume that $n \geq 2$. For all $j: 1 \leq j \leq n$, properly color $x_{n-j}$ and color $t_{n-j}$ by Lemma 1, or properly if possible. Then since $x_{1}$ was properly colored, and $t_{1}$ was colored after $x_{1}$, either $x_{1}$ remains properly colored, or $t_{1}$ has the same color as $x_{1}$. Also, we know that $T_{1}$ must be a $(4,4,4)$-face, so by the previous case, we can extend the coloring to $T_{0}$ and get a coloring of $G$, a contradiction.

Lemma 8. There is no $\left(T_{0}, \ldots, T_{n}\right)$-chain so that $(i) n \geq 1$ and $T_{n}$ is a special $(4,4,4)$-face or (ii) $n \geq 2$ and $T_{n}$ is a $(3,4, k)$-face or (iii) $n=1$ and $T_{n}$ is a $\left(3,4,4^{-}\right)$-face.

Proof. Let $T_{0}=u x_{0} t_{0}$ be a $(3,4,4)$-face with $d(u)=3$.

(i) Let $v$ be a special 4 -vertex of $T_{n}$ and let $y$ and $z$ be the neighbors of $v$ other than $t_{n}$ and $x_{n}$. Let $S=\left\{t_{i}, x_{i}: 0 \leq i \leq n-1\right\}$. By the minimality of $G, G \backslash\left(S \cup\left\{u, v, x_{n}, y, z\right\}\right)$ has a $(1,1,0)$-coloring. Properly color $x_{n}, y$ and $z$, then by Lemma 1 color $v$. Then, either $x_{n}$ remains properly colored or $v$ shares the same color, so by Lemma 7 we can extend the coloring to $\left\{T_{0}, T_{1}, \cdots, T_{n-1}\right\}$ to obtain a coloring of $G$.

(ii) Let $v$ be the 3-vertex of $T_{n}$ and let $S=\left\{t_{i}, x_{i}: 0 \leq i \leq n-1\right\}$. By the minimality of $G, G \backslash(S \cup\{u, v\})$ has a $(1,1,0)$ coloring. Properly color $v$ and $x_{n-1}$. Then by Lemma 1 , we can color $t_{n-1}$. Either $x_{n-1}$ remains properly colored or $t_{n-1}$ shares the same color, so by Lemma 7 we can extend the coloring to $\left\{T_{0}, T_{1}, \cdots, T_{n-2}\right\}$ to obtain a coloring of $G$.

(iii) Assume that $n=1$ and $T_{n}$ is a $(3,4,4)$-face with 3 -vertex $v$. By the minimality of $G, G \backslash\left\{t_{0}, u, v, x_{0}, x_{1}\right\}$ has a $(1,1,0)$-coloring. Properly color $x_{0}$ and $u$ in that order and properly color $x_{1}$ and $v$ in that order. Then $t_{0}$ has four neighbors colored, all properly, so by Lemma 1 we can color $t_{0}$ to get a coloring for $G$.

Remark: By above lemma, a $\left(T_{0}, T_{1}\right)$-chain with $T_{1}$ being a $\left(3,4,5^{+}\right)$-face is not necessarily reducible. Let a bad $\left(3,4,5^{+}\right)$-face be a $\left(3,4,5^{+}\right)$-face that shares a 4 -vertex with a $(3,4,4)$-face.

Lemma 9. There is no $\left(T_{0}, \ldots, T_{n}\right)$-chain with $T_{i}=T_{n}$ for some $i \neq n$.

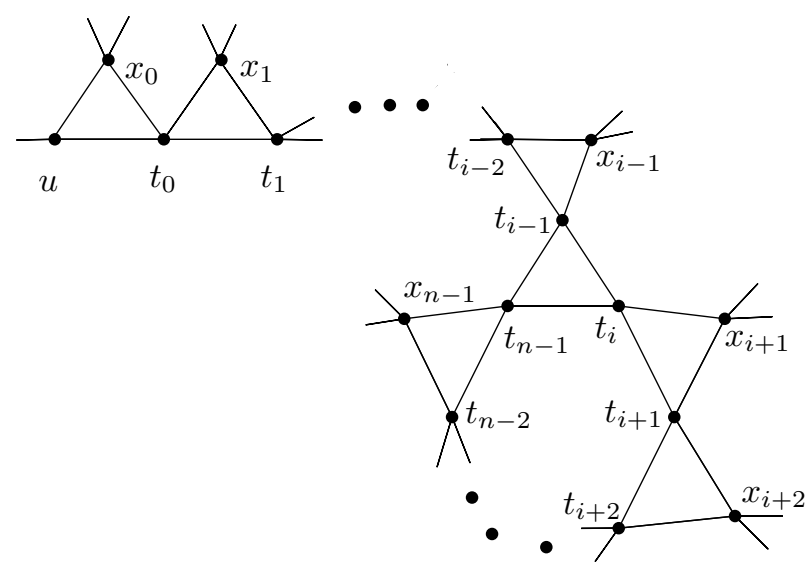

Figure 3. Figure for Lemma 9 
Proof. Let $\left(T_{0}, \ldots, T_{n}\right)$-chain be a chain with $T_{i}=T_{n}$ for some $i<n$. Let $u$ be the 3 vertex of $T_{0}$ and let $S=\left\{t_{j}, x_{j}: 0 \leq j \leq n-1\right\}$. Since $T_{i}=T_{n}$, the vertex that would have been labelled $x_{i}$ is instead labelled $t_{n-1}$ (See Figure 3). By the minimality of $G$, $G \backslash(S \cup\{u\})$ is $(1,1,0)$-colorable. Start by properly coloring $x_{i+1}, x_{i+2}$, and $t_{i+1}$. Then for all $j: i+2 \leq j \leq n-2$, properly color $x_{j+1}$ and color $t_{j}$ by Lemma 1. Next, properly color $t_{n-1}$, and we have two cases:

Case 1: $i=0$. We can properly color $u$, then color $t_{i}$ by Lemma 1 to get a coloring of $G$, a contradiction.

Case 2: $i>0$. We can then color $t_{i}$ by Lemma 1 and then either $t_{n-1}$ is properly colored, or $t_{i}$ shares the same color, so by Lemma 7 we can extend the coloring to $\left\{T_{0}, T_{1}, \cdots, T_{i-1}\right\}$ to obtain a coloring of $G$, a contradiction.

Lemma 10. For each $(3,4,4)$-face $T_{0}$ without good 4-vertices, there exist two chains, $\left(T_{0}, \ldots, T_{n}\right)$ chain and $\left(T_{0}, \ldots, T_{m}^{\prime}\right)$-chain, such that $T_{n}$ and $T_{m}^{\prime}$ are either bad $\left(3,4,5^{+}\right)$-faces, $\left(4,4^{+}, 5^{+}\right)$faces, or $(4,4,4)$-faces with a good 4-vertex. Furthermore, $T_{n} \neq T_{m}^{\prime}$.

Proof. As $G$ is finite, any chain of triangles in $G$ must be finite. By Lemma 8 and 9 , no chain of triangles in $G$ can end with a special 3 -face or a non-bad $\left(3,4,5^{+}\right)$-face, thus it must end with a bad $\left(3,4,5^{+}\right)$-face or a $\left(4,4^{+}, 4^{+}\right)$-face. Since a $(4,4,4)$-face in a chain can not be a special 3 -face, any chain of triangles in $G$ must end with a bad $\left(3,4,5^{+}\right)$-face, a $\left(4,4^{+}, 5^{+}\right)$-face or a $(4,4,4)$-face with a good 4 -vertex.

Now we assume that $T_{n}=T_{m}$. Then by Lemma $7, T_{n}$ must be a $\left(4,4,5^{+}\right)$-face, and since $G$ has no 4 - and 5-cycles, $n+m \geq 6$. Assume that $n \leq m$. Let $S=\left\{t_{i}, x_{i}: 1 \leq i \leq n-1\right\}$, where $S=\emptyset$ if $n=1$, and $S^{\prime}=\left\{t_{j}^{\prime}, x_{j}^{\prime}: 0 \leq j \leq m-1\right\}$ and let $u$ be the 3 -vertex of $T_{0}$. By the minimality of $G, G \backslash S \cup S^{\prime} \cup\{u\}$ has a $(1,1,0)$-coloring. We have two cases:

If $n=1$, properly color $x_{m-1}^{\prime}$ and $t_{m-1}^{\prime}$. Then, by Lemma 7 we can extend the coloring to $\left\{T_{0}, T_{1}^{\prime}, \cdots T_{m-2}^{\prime}\right\}$ to obtain a coloring of $G$, a contradiction.

If $n \geq 2$, then properly color $x_{n-1}, t_{n-1}$ and $x_{m-1}^{\prime}$ in that order, then by Lemma 1 we can color $t_{m-1}^{\prime}$. If $n \geq 3$, for all $i: 2 \leq i \leq n-1$, properly color $x_{n-i}$ and by Lemma 1 we can color $t_{n-1}$. Then since either $x_{m-1}^{\prime}$ is still properly colored or shares the same color as $t_{m-1}^{\prime}$, by Lemma 7 we can extend the coloring to $\left\{T_{0}, T_{1}^{\prime}, \cdots, T_{m-2}^{\prime}\right\}$ to obtain a coloring of $G$, a contradiction.

We will now prove some lemmas which will ensure that bad $\left(3,4,5^{+}\right)$-faces will have extra charge to help $(3,4,4)$-faces.

Lemma 11. A 5-vertex incident to a bad (3,4,5)-face cannot be incident to another bad $(3,4,5)$-face or a $(3,3,5)$-face.

Proof. We only show the case when a 5-vertex $v$ is incident to two bad $(3,4,5)$-faces, and it is very similar (and easier!) to show the case when it is incident to a bad $(3,4,5)$-face and a $(3,3,5)$-face.

Let $v$ be a 5 -vertex that is incident two bad $(3,4,5)$-faces, $f_{1}$ and $f_{2}$, and let $u$ be a $k$-vertex adjacent $v$ (see Figure 4 ). Let $f_{3}$ be the $(3,4,4)$-face sharing a 4 -vertex with $f_{1}$ and let $f_{4}$ be the $(3,4,4)$-face sharing a 4 -vertex with $f_{2}$. Let $f_{3}$ and $f_{4}$ have outer 4 -vertices of $x$ and $x^{\prime}$ respectively and 3 -vertices of $y$ and $y^{\prime}$ respectively. Also, let $f_{1}$ and $f_{2}$ have 4 -vertices $z$ and $z^{\prime}$. Then, by the minimality of $G, G \backslash\left\{f_{1}, f_{2}, f_{3}, f_{4}\right\}$ has a $(1,1,0)$-coloring. 


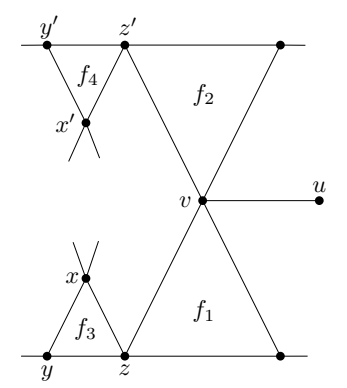

FIgURE 4. Figure for Lemma 11

If $u$ is colored by 1 or 2 , then we can color $v$ by 3 and color the 3 -vertices of $f_{1}$ and $f_{2}$ properly. Since $v$ is properly colored, by Lemma 7 we can extend the coloring to $f_{1}$ and $f_{3}$. Then, since $v$ is colored by 3 , it would remain properly colored, so again by Lemma 7 we can extend the coloring to $f_{2}$ and $f_{4}$ to get a coloring of $G$.

If $u$ is colored by 3 , then we properly color $x$ and $x^{\prime}$ then properly color $y$ and $y^{\prime}$. We then properly color $z$ and $z^{\prime}$. If either $z$ or $z^{\prime}$ is colored by 3 , then we can properly color the 3 -vertices of $f_{1}$ and $f_{2}$ and color $v$ by either 1 or 2 getting a coloring for $G$. So we can assume neither is colored by 3 , and w.l.o.g. we can assume that $z$ is colored by 1 . Then since $z$ and $z^{\prime}$ are properly colored, we can color the 3 -vertices of $f_{1}$ and $f_{2}$ by either 1 or 3 . Then since $v$ will have at most one neighbor colored by 2 , and that neighbor colored properly, we can color $v$ by 2 to obtain a coloring for $G$.

Lemma 12. A $(3,5, k)$-face in $G$ that is incident a 5 -vertex that is also incident to a bad $(3,4,5)$-face and a pendant $\left(3,4^{-}, 4^{-}\right)$-face will have a pendant neighbor that is a $4^{+}$-vertex.

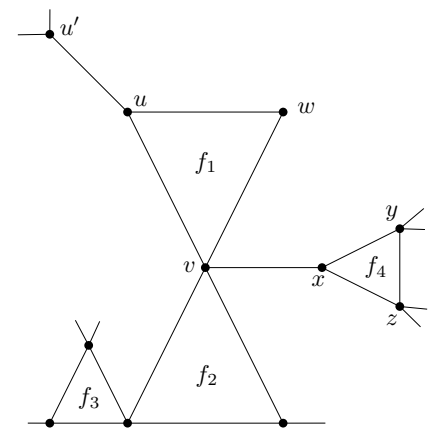

FiguRE 5. Figure for Lemma 12

Proof. Let $f_{1}$ be a $(3,5, k)$-face in $G$ with a 5 -vertex $v$, a 3 -vertex $u$, and a pendant neighbor $u^{\prime}$ that is a 3 -vertex. Let the $k$-vertex of $f_{1}$ be $w$. Let $v$ be incident a bad $(3,4,5)$-face $f_{2}$ with neighbor $(3,4,4)$-face $f_{3}$, and let $v$ have a pendant $(3,4,4)$-face $f_{4}$. Let the 3 -vertex of $f_{4}$ be $x$ and the 4-vertices of $f_{4}$ be $y$ and $z$ (See Figure 5). By the minimality of $G$, $G \backslash\left\{f_{2}, f_{3}, u, u^{\prime}, x\right\}$ has a $(1,1,0)$-coloring. Properly color $x$. If $w$ and $x$ share the same color, then we can properly color $u^{\prime}$ and $u$, then properly color $v$ and the 3 -vertex of $f_{2}$. Then the coloring can be extended to $f_{3}$ by Lemma 7, obtaining a coloring of $G$. So we can assume that $w$ and $x$ are colored differently. If $x$ is colored by 1 or 2 (w.l.o.g. we may assume that 
$x$ is colored by 1 ), then we can color $u^{\prime}$ properly and color $u$ by 1 . Then we can properly color $v$ and properly color the 3 -vertex of $f_{2}$. Finally we can apply Lemma 7 to extend the coloring to $f_{3}$, obtaining a coloring of $G$. So we can assume that $x$ is colored by 3 .

Since $x$ is colored by 3, we may assume that $w$ is colored by 1 . Properly color $u^{\prime}$ and color $u$ by 2 . Since $x$ is properly colored, $y$ and $z$ must be colored by 1 and 2 . W.l.o.g. let $y$ be colored by 1 . Then to avoid being able to re-color $x$ by 1 , the two other neighbors of $y$ must be colored 1 and 3. For similar reasons the other two neighbors of $z$ must be colored 2 and 3. Then switch the colors of $y$ and $z$ and color $x$ by 1 or 2 and color $v$ by 3, we can color the 3 -vertex of $f_{2}$ properly and by Lemma 7, extend the coloring to $f_{3}$, obtaining a coloring of $G$.

Lemma 13. $A(3,5,5)$-face in $G$ can not have both 5 -vertices also be incident to bad $(3,4,5)$ faces and have pendant $(3,4,4)$-faces.

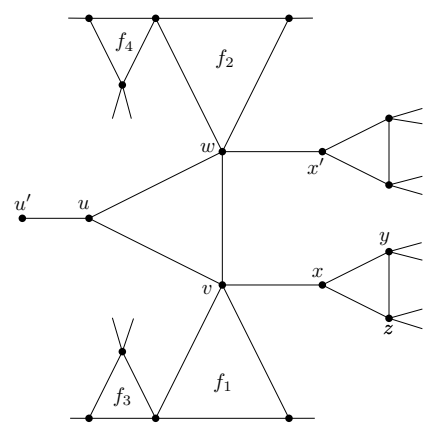

FiguRE 6. Figure for Lemma 13

Proof. Let $u v w$ be a $(3,5,5)$-face in $G$ where $d(v)=d(w)=5$ and $u$ has pendant neighbor $u^{\prime}$. Also let $v$ and $w$ both be incident bad $(3,4,5)$-faces, $f_{1}$ and $f_{2}$ with neighbor $(3,4,4)$-faces $f_{3}$ and $f_{4}$ respectively and let $v$ and $w$ have pendant $(3,4,4)$-faces. Let the pendant $(3,4,4)$ faces to $v$ and $w$ have 3-vertices $x$ and $x^{\prime}$ respectively (See Figure 6). By the minimality of $G, G \backslash\left\{f_{1}, f_{2}, f_{3}, f_{4}, u, x, x^{\prime}\right\}$ has a $(1,1,0)$-coloring.

Properly color $x$ and $x^{\prime}$. If either $x$ or $x^{\prime}$ has a coloring different from $u^{\prime}$, w.l.o.g. we can assume $x$, then we color $u$ the same as $x$. We can properly color $w$ and $v$ in that order, then properly color the 3 -vertices of $f_{1}$ and $f_{2}$. Then by Lemma 7 we can extend the coloring to $f_{3}$ and $f_{4}$ to obtain a coloring of $G$. So we can assume that $x, x^{\prime}$, and $u^{\prime}$ are colored the same. If $x$ is colored by 3 , since $x$ is properly colored, $y$ and $z$ must be colored by 1 and 2 . Then to avoid being able to re-color $x$ by 1 , the other two neighbors of $y$ must be colored 1 and 3. For similar reasons the other two neighbors of $z$ must be colored 2 and 3 . Then we can switch the colors of $y$ and $z$ and color $x$ differently from $u^{\prime}$. Then we follow the above procedure to obtain a coloring for $G$.

So we may assume that w.l.o.g. $x, x^{\prime}$, and $u^{\prime}$ are all colored by 1 . Then we color $u$ by 2 and $w$ by 3 . Color the 3 -vertex of $f_{2}$ properly and by Lemma 7, extend the coloring to $f_{4}$. We now have $v$ adjacent to 3 differently and properly colored vertices. Properly color the outer 4 -vertex and the 3 -vertex of $f_{3}$ in that order, then properly color the 4 -vertex of $f_{1}$. If it is colored by 3 , then properly color the 3 -vertex of $f_{1}$ and color $v$ by either 1 or 2 to obtain a coloring of $G$. If it is not colored by 3 , then w.l.o.g. we can assume that it is colored by 1 . 
Then since it is properly colored, we can color the 3 -vertex of $f_{1}$ by either 1 or 3 and color $v$ by 2 , obtaining a coloring of $G$.

Lemma 14. A 5-vertex in $G$ that is incident a bad $(3,4,5)$-face and has a pendant $(3,4,4)$ face cannot also be incident a $\left(4,4^{+}, 5\right)$-face $T_{n}$ that is in a $\left(T_{0}, \ldots, T_{n}\right)$-chain.

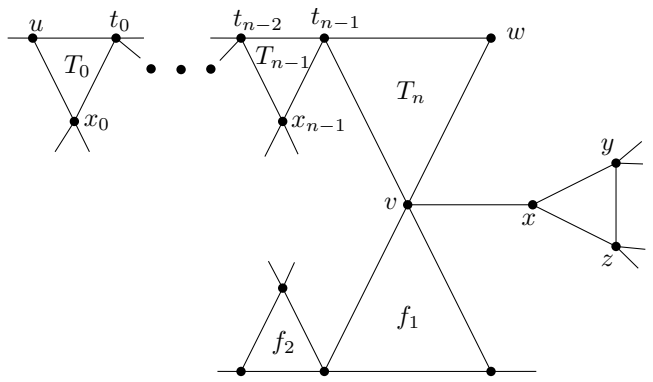

Figure 7. Figure for Lemma 14

Proof. Let $v$ be a 5 -vertex in $G$ that is incident a bad $(3,4,5)$-face $f_{1}$ with neighbor $(3,4,4)$ face $f_{2}$. Let $v$ have a pendant $(3,4,4)$-face with 3 -vertex $w$ and 4 -vertices $y$ and $z$. Also let $v$ be incident a $\left(4,4^{+}, 5\right)$-face $T_{n}$ such that there exists a chain of triangles from $T_{0}$ to $T_{n}$. Let the $4^{+}$-vertex of $T_{n}$ be $w$. Let $S=\left\{t_{i}, x_{i}: 0 \leq i \leq n-1\right\}$ and let $u$ be the 3-vertex of $T_{0}$ (See Figure 7). By the minimality of $G, G \backslash\left(S \cup\left\{f_{1}, f_{2}, u, x\right\}\right)$ has a $(1,1,0)$-coloring.

Properly color $x$. If $x$ and $w$ are colored the same then we can properly color $x_{n-1}, t_{n-1}$, and $v$. If $n=1$, then by Lemma 1 , we can color $u$. If $n \geq 2$, then by Lemma 7 we can extend the coloring to $\left\{T_{0}, T_{1}, \cdots, T_{n-1}\right\}$. Then we can properly color the 3 -vertex of $f_{1}$ and by Lemma 7 we can extend the coloring to $f_{2}$ obtaining a coloring for $G$. So we can assume that $x$ and $w$ are colored differently.

Let $x$ be colored 1 or 2 and w.l.o.g. we can assume that $x$ is colored by 1 . Then we can properly color $x_{n-1}$ and color $t_{n-1}$ by 1 . Since $w$ and $x$ are colored differently, either $x_{n-1}$ and $t_{n-1}$ are both colored properly or share the same color. If $n=1$, then either we can color $u$ properly or we can color $u$ by Lemma 1 . If $n \geq 2$, then by Lemma 7 we can extend the coloring to $\left\{T_{0}, T_{1}, \cdots, T_{n-1}\right\}$. Then since $t_{n-1}$ and $x$ are colored the same we can properly color $v$ and the 3 -vertex of $f_{1}$. By Lemma 7 we can extend the coloring to $f_{2}$ to obtain a coloring of $G$.

So let $x$ be colored by 3 (then $w$ is colored 1 or 2 ). Then $y$ and $z$ must be colored by 1 and 2 , respectively. To avoid being able to re-color $x$ by 1 or 2 , the two other neighbors of $y$ must be colored 1 and 3 and the two other neighbors of $z$ must be colored 2 and 3 . Then we switch the colors of $y$ and $z$ and re-color $x$ to be the same as $w$, and proceed as above to get a coloring for $G$.

Lemma 15. Every 6-vertex in $G$ that is incident a bad $(3,4,6)$-face can be incident at most two $\left(3,4^{-}, 6\right)$-faces.

Proof. Let $v$ be a 6 -vertex in $G$. Let $v w x$ be a bad $(3,4,6)$-face with $d(w)=3$ and neighbor $(3,4,4)$-face $x y z$ with 3 -vertex $y$. Let $v$ also be incident non-bad $(3,4,6)$-faces $t_{1} t_{2} v$ and $u_{1} u_{2} v$ 


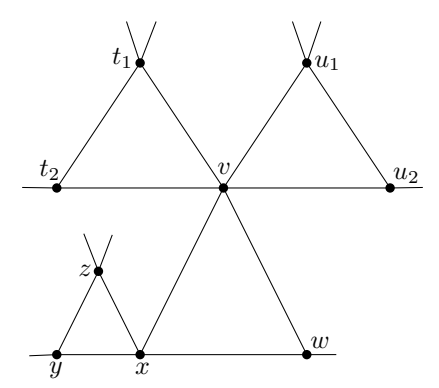

Figure 8. Figure for Lemma 15

where $d\left(t_{1}\right)=d\left(u_{1}\right)=4$ (See Figure 8). By the minimality of $G, G \backslash\left\{t_{1}, t_{2}, u_{1}, u_{2}, v, w, x, y, z\right\}$ has a $(1,1,0)$-coloring. Properly color $t_{1}, t_{2}, u_{1}$, and $u_{2}$. If the color set of $\left\{t_{1}, t_{2}, u_{1}, u_{2}\right\}$ is not $\{1,2,3\}$, then we can properly color $v$ and $w$. Then by Lemma 7, we can extend the coloring to $x, y$, and $z$, obtaining a coloring of $G$. So we can assume that the color set of $\left\{t_{1}, t_{2}, u_{1}, u_{2}\right\}$ includes 1,2 , and 3 .

If two of $\left\{t_{1}, t_{2}, u_{1}, u_{2}\right\}$ are colored by 3 , then we can color $z, y$, and $x$ properly. If $x$ is colored by 3 , then we can color $w$ properly and color $v$ by 1 or 2 to get a coloring of $G$. If $x$ is colored by 1 or 2 , then since $x$ is properly colored we can color $w$ by 3 or the same as $x$. Then we can color $v$ differently from 3 and $x$ to obtain a coloring of $G$.

So we can assume that exactly one of vertices in the set $\left\{t_{1}, t_{2}, u_{1}, u_{2}\right\}$ is colored by 3 . Then w.l.o.g. we may assume that the color set of $\left\{t_{1}, t_{2}\right\}$ is $\{1,3\}$ and the color set of $\left\{u_{1}, u_{2}\right\}$ is $\{1,2\}$. Since $u_{1}$ and $u_{2}$ were colored properly, the outside neighbor of $u_{2}$ must be 3 . Let $u_{1}$ be colored by 1 , then since it is colored properly we can recolor $u_{2}$ by 1 . Then we can color $v$ and $w$ properly, and extend to $x, y$, and $z$ to obtain a coloring of $G$. So we can assume that $u_{1}$ is colored by 2 .

Now color $z, y$, and $x$ properly in that order. If $x$ is colored by 3 then color $w$ properly. If $w$ is colored by 1 , then color $v$ by 2 to get a coloring for $G$. If $w$ is colored by 2 , then since $u_{1}$ is colored properly recolor $u_{2}$ by 2 and color $v$ by 1 to get a coloring for $G$. So we can assume that $x$ is colored by 1 or 2 . Since $x$ is properly colored we can color $w$ by 3 or the same as $x$. Then either 1 or 2 but not both is in the color set of $\{x, w\}$. If 1 is in the color set, then $v$ will have only one neighbor colored by 2 so we can color $v$ by 2 and obtain a coloring of $G$. If 2 is in the color set, then $v$ will have two neighbors colored by 1 , but we can recolor $u_{2}$ by 2 and color $v$ by 1 to obtain a coloring of $G$.

The following lemma says that a 3 -face with $k$ vertices of degree 4 can have at most $k$ chains of triangles ending at it.

Lemma 16. If a $\left(T_{0}, T_{1}, \ldots, T_{n}\right)$-chain and a $\left(T_{0}^{\prime}, T_{1}^{\prime}, \ldots, T_{m}^{\prime}\right)$-chain with $T_{m}^{\prime}=T_{n}$ satisfy $T_{n-1} \cap T_{n}=\left\{t_{n}\right\}=T_{m-1}^{\prime} \cap T_{m}^{\prime}$, then $T_{0}=T_{0}^{\prime}$.

Proof. For otherwise, the two chains have a common $(4,4,4)$-face $T$ so that $T=T_{a}$ and $T=T_{b}^{\prime}$. Then we would have a $\left(T_{0}, T_{1}, T_{a-1}, T, T_{b-1}^{\prime}, \ldots, T_{1}^{\prime}, T_{0}^{\prime}\right)$-chain. But by Lemma 8 , this chain cannot exist in $G$.

\section{Discharging Procedure}


As we mentioned in the introduction, we set the initial charge of a vertex $v$ to be $\mu(v)=2 d(v)-6$ and the initial charge of a face $f$ to be $\mu(f)=d(f)-6$. For the discharging procedure we must introduce the notion of a bank, which serves as a temporary placeholder for charges. We set the bank with initial charge zero and will show it has a non-negative final charge.

The following are the rules for discharging:

(R1) Each 4-vertex gives $\frac{1}{2}$ to each pendant 3 -face and the rest to the incident 3 -faces evenly.

(R2) Every 6-vertex gives $\frac{9}{4}$ to incident bad $(3,4,6)$-faces, 2 to other incident $\left(3,4^{-}, 6\right)$-faces and $\frac{3}{2}$ to all other incident 3 -faces; every $7^{+}$-vertex gives $\frac{9}{4}$ to all incident 3 -faces.

(R3) Every $6^{+}$-vertex gives $\frac{1}{2}$ to all pendant 3 -faces.

(R4) Every $\left(4^{+}, 4^{+}, 5^{+}\right)$-face and every $(4,4,4)$-face with a good 4 -vertex give $\frac{1}{2}$ to the bank and every bad $\left(3,4,5^{+}\right)$-face gives $\frac{1}{4}$ to the bank.

(R5) The bank gives $\frac{1}{2}$ to each $(3,4,4)$-face without good 4 -vertices.

(R6) Every 5-vertex gives

(a) 2 to each incident $(3,3,5)$-face and $9 / 4$ to each incident bad $(3,4,5)$-face.

(b) $7 / 4$ to incident non-bad $(3,4,5)$-faces when also incident a bad $(3,4,5)$-face, and gives 2 to incident non-bad $(3,4,5)$-faces otherwise.

(c) $5 / 4$ to incident $\left(3,5^{+}, 5^{+}\right)$-faces when also incident to a bad $(3,4,5)$-face and a pendant $\left(3,4^{-}, 4^{-}\right)$-face, and gives $3 / 2$ to incident $\left(3,5^{+}, 5^{+}\right)$-faces otherwise.

(d) $3 / 2$ to all $\left(4,4^{+}, 5\right)$-faces with a chain of triangles to a $(3,4,4)$-face and gives 1 to $\left(4,4^{+}, 5\right)$-faces otherwise.

(e) $1 / 2$ to each pendant $\left(3,4^{-}, 4^{-}\right)$-face and $(3,3, k)$-face and $1 / 4$ to all other pendant 3-faces.

Let $v$ be a $k$-vertex. By Proposition 1, $k \geq 3$.

For $k=3$, the final charge $\mu^{*}(v)$ of $v$ is $\mu^{*}(v)=\mu(v)=0$.

For $k=4$, by (R1), the final charge of $v$ is 0 . We note that $v$ gives at least 1 to each incident 3-face, and gives at least $3 / 2$ to 3 -faces when $v$ is a good 4 -vertex.

For $k=5$, if $v$ has at most one incident 3 -face, then by (R6a) and (R6e), $\mu^{*}(v) \geq$ $\mu(v)-\frac{9}{4} \cdot 1-\frac{1}{2} \cdot 3=1 / 4>0$. Let $v$ have two incident 3 -faces $f_{1}$ and $f_{2}$ and a pendant 3 -face $f_{3}$.

Let $f_{3}$ be a $\left(3,4^{-}, 4^{-}\right)$-face. When $f_{1}$ is a bad $(3,4,5)$-face, by Lemma $3 f_{2}$ cannot be a $\left(3,4^{-}, 5\right)$-face. By Lemma 14 , if $f_{2}$ is a $\left(4,4^{+}, 5\right)$-face, then there is no chain of triangles from some $(3,4,4)$-face to $f$, so by (R6a), (R6c), (R6d), and (R6e), $\mu^{*}(v) \geq \mu(v)-\frac{1}{2} \cdot 1-\frac{9}{4} \cdot 1-\frac{5}{4} \cdot 1=$ 0 . When $f_{1}$ is a non-bad $(3,4,5)$-face, then by Lemma $3, f_{2}$ cannot be a $\left(3,4^{-}, 5\right)$-face, so by (R6b), (R6c), (R6d), and (R6e), $\mu^{*}(v) \geq \mu(v)-\frac{1}{2} \cdot 1-2 \cdot 1-\frac{3}{2} \cdot 1=0$. When neither $f_{1}$ nor $f_{2}$ are $\left(3,4^{-}, 5\right)$-faces, by (R6c), (R6d), and (R6e), $\mu^{*}(v) \geq \mu(v)-\frac{1}{2} \cdot 1-\frac{3}{2} \cdot 2=\frac{1}{2}>0$.

Now let $f_{3}$ be a $(3,4,5)$-face. When $f_{1}$ or $f_{2}$ is $\left(3,4^{-}, 5\right)$-face, by Lemma 3 , the other one cannot be a $\left(3,4^{-}, 5\right)$-face, so by (R6b), (R6c), (R6d), and (R6e), $\mu^{*}(v) \geq \mu(v)-\frac{1}{4} \cdot 1-\frac{9}{4}$. $1-\frac{3}{2} \cdot 1=0$. When neither $f_{1}$ nor $f_{2}$ are $\left(3,4^{-}, 5\right)$-faces, by rules (R6c), (R6d), and (R6e), $\mu^{*}(v) \geq \mu(v)-\frac{1}{4} \cdot 1-\frac{3}{2} \cdot 2=\frac{3}{4}>0$.

Finally, let $v$ have two incident 3 -faces $f_{1}$ and $f_{2}$, and no pendant 3 -face. If $f_{1}$ is a bad $(3,4,5)$-face, then by Lemma 11, $f_{2}$ cannot also be a bad $(3,4,5)$-face or a $(3,3,5)$-face. 
Then by $(\mathrm{R} 6), \mu^{*}(v) \geq \mu(v)-\frac{9}{4} \cdot 1-\frac{7}{4} \cdot 1=0$. If neither $f_{1}$ nor $f_{2}$ is a bad $(3,4,5)$-face, then by (R6b), (R6c), and (R6d), $\mu^{*}(v) \geq \mu(v)-2 \cdot 2=0$.

For $k=6$, if $v$ is incident to at most two 3 -faces, then by (R2) and (R3), $\mu^{*}(v) \geq$ $\mu(v)-\frac{9}{4} \cdot 2-\frac{1}{2} \cdot 2=\frac{1}{2}$. So we can assume that $v$ is incident to three 3 -faces. If $v$ is incident a bad $(3,4,6)$-face then by Lemma 15 only one other incident 3 -face can be a $\left(3,4^{-}, 6\right)$-face. So by $(\mathrm{R} 2), \mu^{*}(v) \geq \mu(v)-\frac{9}{4} \cdot 2-\frac{3}{2} \cdot 1=0$. If $v$ is not incident a bad $(3,4,6)$-face, then by $(\mathrm{R} 2), \mu^{*}(v) \geq \mu(v)-2 \cdot 3=0$.

For $k \geq 7$, if $k$ is odd, then $\mu^{*}(v) \geq \mu(v)-\frac{k-1}{2} \cdot \frac{9}{4}-\frac{1}{2} \cdot 1=2 k-6-\frac{9 k-9}{8}-\frac{4}{8}=\frac{7 k-43}{8} \geq \frac{3}{4}$. If $k$ is even, then $\mu^{*}(v) \geq \mu(v)-\frac{k}{2} \cdot \frac{9}{4}=2 k-6-\frac{9 k}{8}=\frac{7 k-48}{8} \geq 1$.

Now let $f$ be a $k$-face. Since $G$ is a simple graph, $k \geq 3$. By the condition that there is no 4 -cycle and 5 -cycle, $k=3$ or $k \geq 6$. Since no faces above degree 3 are involved in the discharging procedure, the final charge of $6^{+}$-face $f$ is $\mu^{*}(f)=\mu(f)=d(f)-6 \geq 0$.

For $k=3$, by Lemma 2 , we have no $\left(3,3,4^{-}\right)$-faces, but we still have a few different cases:

Case 1: Face $f$ is a $\left(3,3,5^{+}\right)$-face. By Lemma 4, $f$ will have two pendant neighbors of degree 4 or higher. So by (R1), (R2), (R4), and (R7), $\mu^{*}(f) \geq(3-6)+2 \cdot 1+\frac{1}{2} \cdot 2=0$.

Case 2: Face $f$ is a $(3,4,4)$-face. By Lemma 5, $f$ will have a pendant neighbor of degree 4 or higher. If $f$ has a good 4 -vertex, then by (R1), $\mu^{*}(f) \geq \mu(f)+\frac{3}{2} \cdot 1+1 \cdot 1+\frac{1}{2} \cdot 1=0$. If $f$ has no good 4 -vertices, then by $(\mathrm{R} 5), f$ receives $1 / 2$ from the bank, so $\mu^{*}(f)=\mu(f)+$ $1 \cdot 2+\frac{1}{2} \cdot 1+\frac{1}{2}=0$.

Case 3: Face $f$ is a bad $(3,4,5)$-face. By (R1), (R4) and (R6a), $\mu^{*}(f)=\mu(f)+1$. $1+\frac{9}{4} \cdot 1-\frac{1}{4} \cdot 1=0$.

Case 4: Face $f$ is a non-bad $(3,4,5)$-face. If the 5 -vertex of $f$ is not incident a bad $(3,4,5)$-face, then by $(\mathrm{R} 1)$ and $(\mathrm{R} 6 \mathrm{~b}), \mu^{*}(f)=\mu(f)+1 \cdot 1+2 \cdot 1=0$. If the 5 -vertex of $f$ is incident a bad $(3,4,5)$-face, then by Lemma $12, f$ has a pendant neighbor of degree 4 or higher. So by (R1), (R6b), and (R6e), $\mu^{*}(f) \geq \mu(f)+1 \cdot 1+\frac{7}{4} \cdot 1+\frac{1}{4} \cdot 1=0$.

Case 5: Face $f$ is a $(3,4,6)$-face. If $f$ is a bad $(3,4,6)$-face, then by (R1), (R2), and (R4), $\mu^{*}(f)=\mu(f)+1 \cdot 1+\frac{9}{4} \cdot 1-\frac{1}{4} \cdot 1=0$. If $f$ is a non-bad $(3,4,6)$-face then by (R1) and (R2), $\mu^{*}(f)=\mu(f)+1 \cdot 1+2 \cdot 1=0$.

Case 6: Face $f$ is a $\left(3,4,7^{+}\right)$-face. By (R1) and (R2), $\mu^{*}(f)=\mu(f)+1 \cdot 1+\frac{9}{4} \cdot 1=\frac{1}{4}$.

Case 7: Face $f$ is a $(3,5,5)$-face. If neither 5-vertex of $f$ is also incident to a bad $(3,4,5)$-face and a pendant $\left(3,4^{-}, 4^{-}\right)$-face, then by (R6c), $\mu^{*}(f)=\mu(f)+\frac{3}{2} \cdot 2=0$. If one of the 5 -vertices of $f$ is also incident to a bad $(3,4,5)$-face and a pendant $\left(3,4^{-}, 4^{-}\right)$-face then by Lemma 12, $f$ must have a pendant neighbor of degree 4 or higher. In addition, by Lemma 13 the other 5 -vertex of $f$ cannot have both an incident bad $(3,4,5)$-face and a pendant $\left(3,4^{-}, 4^{-}\right)$-face. So by (R6c) and (R6e), $\mu^{*}(f)=\mu(f)+\frac{5}{4} \cdot 1+\frac{1}{4} \cdot 1+\frac{3}{2} \cdot 1=0$.

Case 8: Face $f$ is a $\left(3,5,6^{+}\right)$-face. If the 5 -vertex of $f$ is not incident to a bad $(3,4,5)$ face and a pendant $\left(3,4^{-}, 4^{-}\right)$-face then by (R2) and (R6c), $\mu^{*}(f) \geq \mu(f)+\frac{3}{2} \cdot 2=0$. If the 5 -vertex of $f$ has both an incident bad $(3,4,5)$-face and a pendant $\left(3,4^{-}, 4^{-}\right)$-face, then by Lemma $12 f$ must have a pendant neighbor of degree 4 or higher. So by (R2), (R6c), and (R6e), $\mu^{*}(f) \geq \mu(f)+\frac{5}{4} \cdot 1+\frac{1}{4} \cdot 1+\frac{3}{2} \cdot 1=0$. 
Case 9: Face $f$ is a $\left(3,6^{+}, 6^{+}\right)$-face. By $(\mathrm{R} 2), \mu^{*}(f) \geq \mu(f)+\frac{3}{2} \cdot 2=0$.

Case 10: Face $f$ is a $(4,4,4)$-face. If $f$ has no good 4 -vertices then by $(\mathrm{R} 1), \mu^{*}(f)=$ $\mu(f)+1 \cdot 3=0$. If $f$ has a good 4 -vertex then by $(\mathrm{R} 1)$ and $(\mathrm{R} 4), \mu^{*}(f) \geq \mu(f)+1 \cdot 2+\frac{3}{2} \cdot 1-\frac{1}{2} \cdot 1=$ 0 .

Case 11: Face $f$ is a $\left(4^{+}, 4^{+}, 5^{+}\right)$-face. If $f$ has no chains of triangles to a $(3,4,4)$-face, then each incident vertex gives at least 1 to $f$, so $\mu^{*}(f) \geq \mu(f)+1 \cdot 3=0$. If $f$ has a chain of triangles to a $(3,4,4)$-face then by (R6d), at least one vertex must give $\frac{3}{2}$ to $f$, so combined with $(\mathrm{R} 4), \mu^{*}(v) \geq \mu(v)+1 \cdot 2+\frac{3}{2} \cdot 1-\frac{1}{2} \cdot 1=0$.

Finally, we show that the bank has a non-negative charge. By Lemma 10 , for each $(3,4,4)$ face without good 4 -vertices in $G$, there exist at least two chains of triangles from the $(3,4,4)$ face to a bad $\left(3,4,5^{+}\right)$-face, a $(4,4,4)$-face with a good 4 -vertex, or a $\left(4^{+}, 4^{+}, 5^{+}\right)$-face. Then by Lemma 16, there exist at most two chains of triangles to $\left(4^{+}, 4^{+}, 5^{+}\right)$-face from $(3,4,4)$ faces and at most one chain of triangles to a $\left(3,4,5^{+}\right)$-face from $(3,4,4)$-faces. So we can see the transfer of charge from triangles with extra charge to the bank and back to $(3,4,4)$ faces is a transfer of $\frac{1}{4}$ charge over each chain of triangles. Each $(4,4,4)$-face with a good 4 -vertex and $\left(4^{+}, 4^{+}, 5^{+}\right)$-face gives $\frac{1}{2}$ to the bank, and the bank will give at most $\frac{1}{4} \cdot 2$ to $(3,4,4)$-faces for each $(4,4,4)$-face with a good 4 -vertex or $\left(4^{+}, 4^{+}, 5^{+}\right)$-face. Also, each bad $\left(3,4,5^{+}\right)$-face gives $\frac{1}{4}$ to the bank, and the bank will give at most $\frac{1}{4} \cdot 1$ to $(3,4,4)$-faces for each bad $\left(3,4,5^{+}\right)$-face. Hence the bank will always have a non-negative charge.

This completes the discharging, showing that the final charges of all faces, vertices, and the bank are non-negative, a contradiction to (1). This completes the proof of Theorem 1.1.

\section{3. (3, 0,0)-COLORING OF PLANAR GRAPHS}

In this section, we give a proof for Theorem 2, Our proof will again use a discharging method. Let $G$ be a minimum counterexample to Theorem 2 , that is, $G$ is a planar graph without 4-cycles and 5-cycles and is not $(3,0,0)$-colorable, but any proper subgraph of $G$ is properly $(3,0,0)$-colorable. We may assume that vertices colored by 1 may have up to three neighbors colored by 1 .

The following is a very useful tool to extend a coloring on a subgraph of $G$ to include more vertices.

Lemma 17. Let $H$ be a proper subgraph of $G$. Given a $(3,0,0)$-coloring of $G-H$, if two neighbors of $v \in H$ are colored so that one is a $5^{-}$-vertex and the other is nicely colored, then the coloring can be extended to $G-(H-v)$ such that $v$ is nicely colored by 1 .

Proof. Let $H$ be a subgraph of $G$ such that $G-H$ has a $(3,0,0)$-coloring. Let $v \in H$ have neighbors $u$ and $w$ that are colored. Let $d(u) \leq 5$ and let $w$ be nicely colored. Color $v$ by 1 . Since $w$ is nicely colored, if this coloring is invalid, then $u$ must be colored by 1 . In addition, $u$ must have at least 3 neighbors colored by 1 . To avoid recoloring $u$ by 2 or $3, u$ must have at least one neighbor of color 2 and at least one neighbor of color 3 . This implies that $d(u) \geq 6>5$, a contradiction. So $v$ is colorable by 1 . In addition, since the deficiency of color 1 is 3 and $v$ only has 2 neighbors, it follows that $v$ is nicely colored.

Lemma 18. Every 3-vertex in $G$ has a $6^{+}$-vertex as a neighbor. 
Proof. Let $v$ be a vertex in $G$ such that each neighbor vertex of $v$ has degree 5 . By the minimality of $G, G-v$ is $(3,0,0)$-colorable. If two vertices in $N(v)$ share the same color, then $v$ can be properly colored, so we can assume all the neighbors of $v$ are colored differently. Let $u$ be the neighbor of $v$ that is colored by 1 . Then $u$ must have 3 neighbors colored by 1 to forbid $v$ to be colored by 1 . In addition, $u$ must have neighbors colored by 2 and 3 to forbid $v$ to be colored by 2 or 3 . Then, $u$ has at least 6 neighbors, a contradiction.

Let a $\left(3,3,3^{+}\right)$-face to be poor if the pendant neighbors of the two 3 -vertices have degrees at most 5 . A $\left(3,3^{+}, 3^{+}\right)$-face is semi-poor if exactly one of the pendant neighbors of the 3 -vertices has degree 5 or less. A 3-face is non-poor if each 3-vertex on it has the pendant neighbor being a $6^{+}$-vertex. Finally, a poor 3 -vertex is a 3 -vertex on a poor or semi-poor 3 -face that has a $5^{-}$-vertex as its pendant neighbor.

Lemma 19. All (3,3, $\left.6^{-}\right)$-faces in G are non-poor.

Proof. For all $\left(3,3,5^{-}\right)$-faces in $G$, the proof is trivial by Lemma 18 . Let uvw be a $(3,3,6)$ face in $G$ with $d(u)=d(v)=3$ such that the pendant neighbor $v^{\prime}$ of $v$ has degree at most 5. By the minimality of $G, G \backslash\{u, v\}$ is $(3,0,0)$-colorable. Properly color $u$ and color $v$ differently than both $w$ and $v^{\prime}$. Then $u$ and $v$ are both colored by 2 or 3, w.l.o.g. assume 2. This means that $u^{\prime}$ and $v^{\prime}$ share the same color (where $u^{\prime}$ is the pendant neighbor of $u$ ), different from the color of $w$.

Let $w$ be colored by 1 , then to avoid being able to recolor $u$ or $v$ by $1, w$ must have 3 outer neighbors colored by 1 . Then $w$ can be recolored by 2 or 3 depending on the color of its fourth colored neighbor. We recolor $w$ by 2 or 3 and recolor $u$ and $v$ by 1 to get a coloring of $G$, a contradiction.

So we may assume that $w$ is colored by 3 , and that $u^{\prime}$ and $v^{\prime}$ are colored by 1 . To avoid recoloring $v$ by $1, v^{\prime}$ must have at least 3 neighbors colored by 1 . In addition, to avoid recoloring $v^{\prime}$ by 2 or 3 and coloring $v$ by $1, v^{\prime}$ must have neighbors colored by both 2 and 3 . This contradicts that $v^{\prime}$ has degree less than 6 .

Lemma 20. No vertex $v \in V(G)$ can have $\left\lfloor\frac{d(v)}{2}\right\rfloor$ incident poor 3-faces.

Proof. Let $v$ be a $k$-vertex in $G$ with $\left\lfloor\frac{k}{2}\right\rfloor$ incident poor $(3,3, k)$-faces. Let $u_{1}, u_{2}, \cdots, u_{k}$ be the neighbors of $v$, and let $u_{i}^{\prime}$ be the pendant neighbor if $u_{i}$ is in a poor 3 -face. Note that $d\left(u_{i}^{\prime}\right) \leq 5$ and we know that all except possibly $u_{k}$ are in poor 3-faces.

By the minimality of $G, G \backslash\left\{v, u_{1}, u_{2}, \cdots, u_{k-1}\right\}$ is $(3,0,0)$-colorable. If $d(v)$ is odd, then by Lemma 17, for all $i$ with $1 \leq i \leq k-1$, we can color $u_{i}$ by 1 . Then we can properly color $v$ to get a coloring of $G$, so we can assume that $d(v)$ is even. If $d(v)$ is even, then by Lemma 17, for all $i$ with $1 \leq i \leq k-2$, we can color $u_{i}$ by 2 . Then if $u_{k}$ is colored by 1 we can color $u_{k-1}$ properly and $v$ properly to get a coloring of $G$. If $u_{k}$ is colored by 2 or 3 , then it is colored properly and by Lemma 17 we can color $u_{k-1}$ by 1 . Then we can properly color $v$ to get a coloring of $G$, a contradiction.

Lemma 21. If an 8-vertex $v$ is incident to three incident poor (3,3,8)-faces, then it cannot be incident to a semi-poor face, nor two pendant 3-faces.

Proof. Let $v$ be an 8 -vertex in $G$ with 3 incident poor $(3,3,8)$-faces. Let $u_{1}, u_{2}, \cdots, u_{6}$ be the 3 -vertices in the poor $(3,3,8)$-face and let $u_{1}^{\prime}, u_{2}^{\prime}, \cdots, u_{6}^{\prime}$ be the corresponding pendant neighbors, respectively. We know that for all $i$ with $1 \leq i \leq 6, d\left(u_{i}^{\prime}\right) \leq 5$. 
(i) Let $v u_{7} u_{8}$ be the incident semi-poor face with $u_{7}$ being the poor 3 -vertex. Then by the minimality of $G, G \backslash\left\{v, u_{1}, u_{2}, \cdots, u_{7}\right\}$ is $(3,0,0)$-colorable. By Lemma 17, $u_{1}, u_{2}, \cdots, u_{6}$ can be colored by 1 . Then if $u_{8}$ is colored by 1 , we can properly color $u_{7}$ and then $v$ to get a coloring of $G$. So we may assume that $u_{8}$ is not colored by 1 , in which case it is nicely colored and we may color $u_{7}$ with 1 by Lemma 17, and then properly color $v$ to get a coloring of $G$, a contradiction.

(ii) Let $u_{7}$ and $u_{8}$ be the bad 3 -vertices adjacent to $v$. Then $G \backslash\left\{v, u_{1}, u_{2}, \cdots, u_{7}, u_{8}\right\}$ is $(3,0,0)$-colorable, by the minimality of $G$. Properly color both $u_{7}$ and $u_{8}$. If either $u_{7}$ or $u_{8}$ is colored by 1 or both have the same color, then by Lemma 17 , we may color $u_{1}, u_{2}, \cdots, u_{6}$ by 1 and then properly color $v$. So we may assume that $u_{7}$ is colored by 2 and $u_{8}$ is colored by 3 . Then we properly color $u_{1}, u_{2}, \cdots, u_{6}$, and it follows that for each $i$ with $1 \leq i \leq 3$, $u_{2 i-1}$ and $u_{2 i}$ must be colored differently. Then $v$ can have at most 3 neighbors colored by 1 , all properly colored, so $v$ can be colored by 1 , a contradiction.

Lemma 22. If a 7-vertex $v$ is incident to two poor $(3,3,7)$-faces, then it cannot be (i) incident to a semi-poor $\left(3,6^{-}, 7\right)$-face and adjacent to a pendant 3 -face, or (ii) adjacent to three pendant 3-faces.

Proof. Let $v$ be a 7 -vertex in $G$ with 2 incident poor $(3,3,7)$-faces. Let $u_{1}, u_{2}, u_{3}$, and $u_{4}$ be the 3 -vertices on the poor $(3,3,7)$-faces and let $u_{1}^{\prime}, u_{2}^{\prime}, u_{3}^{\prime}$, and $u_{4}^{\prime}$ be their corresponding pendant neighbors, respectively. We know that for all $i$ with $1 \leq i \leq 4, d\left(u_{i}^{\prime}\right) \leq 5$.

(i) Let $v u_{5} u_{6}$ be a semi-poor face with $u_{5}$ being a poor 3 -vertex and $d\left(u_{6}\right) \leq 6$ and let $u_{7}$ be a bad 3-vertex adjacent to $v$. By the minimality of $G, G \backslash\left\{v, u_{1}, u_{2}, u_{3}, u_{4}, u_{5}, u_{7}\right\}$ is $(3,0,0)$-colorable. Since at this point $u_{6}$ has only 4 colored neighbors, if $u_{6}$ is colored by 1 then either it is nicely colored or it can be recolored properly. If $u_{6}$ is not nicely colored, then recolor $u_{6}$ properly.

Color $u_{7}$ properly. If $u_{7}$ is colored by 1 , then by Lemma 17 , we can color $u_{1}, u_{2}, \cdots, u_{5}$ by 1 and then color $v$ properly, a contradiction. So we may assume w.l.o.g. that $u_{7}$ is colored by 2. Color $u_{1}, u_{2}, \cdots, u_{5}$ properly. Then, for each $i$ with $1 \leq i \leq 3, u_{2 i}$ and $u_{2 i-1}$ are colored differently and nicely. This leaves $v$ with at most 3 neighbors colored by 1 , all nicely, so we may color $v$ by 1 to get a coloring of $G$, a contradiction.

(ii) Let $u_{5}, u_{6}$, and $u_{7}$ be the bad 3 -vertices adjacent to $v$. By the minimality of $G$, $G \backslash\left\{v, u_{1}, \ldots, u_{7}\right\}$ is $(3,0,0)$-colorable. Properly color $u_{5}, u_{6}$, and $u_{7}$. If the set $\left\{u_{5}, u_{6}, u_{7}\right\}$ does not contain both colors 2 and 3 , then by Lemma 17, we can color $u_{1}, u_{2}, u_{3}$, and $u_{4}$ by 1 and color $v$ properly. So we can assume that $\left\{u_{5}, u_{6}, u_{7}\right\}$ contains both colors 2 and 3 . This implies that at most one vertex is colored by 1 . So we properly color $u_{1}, u_{2}, u_{3}$, and $u_{4}$. Then $v$ has at most 3 neighbors colored by 1 , all nicely, so we can color $v$ by 1 to get a coloring of $G$, a contradiction.

Lemma 23. Let uvw be a semi-poor $(3,7,7)$-face in $G$ such that $d(v)=d(w)=7$. Then vertices $v$ and $w$ cannot both be 7-vertices that are incident to two poor 3-faces, one semi-poor $(3,7,7)$-face, and adjacent to one pendant 3 -face.

Proof. Let uvw be a semi-poor $(3,7,7)$-face in $G$ such that $d(v)=d(w)=7$ and both $v$ and $w$ are incident to two poor 3 -faces, one $(3,7,7)$-face, and adjacent to one pendant 3 -face. Let the neighbors of $v$ and $w$ be $t_{1}, t_{2}, \cdots, t_{5}$ and $z_{1}, z_{2}, \cdots, z_{5}$, respectively such that $t_{5}$ and $z_{5}$ are bad 3 -vertices (See Figure 9). 

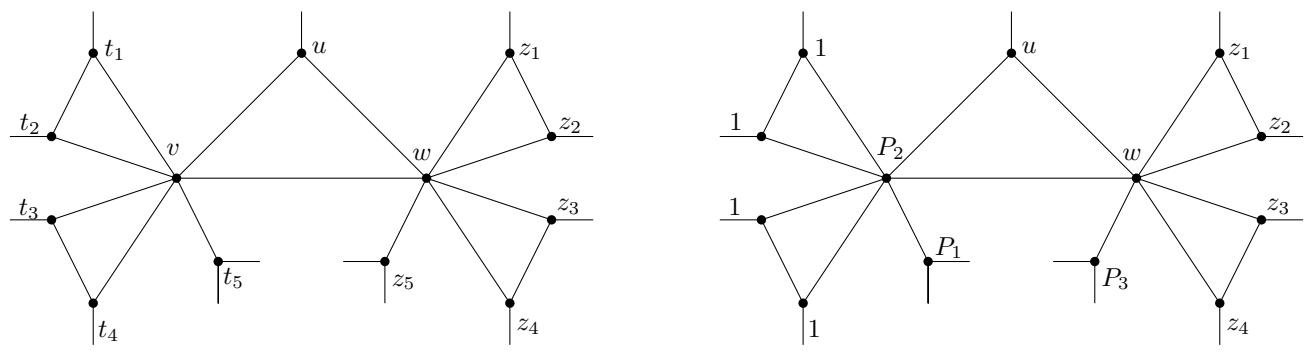

Figure 9. Figure for Lemma 23

By the minimality of $G, G \backslash\left\{u, v, w, t_{1}, t_{2}, \cdots, t_{5}, z_{1}, z_{2}, \cdots, z_{5}\right\}$ is $(3,0,0)$-colorable. By Lemma 17, we can color $t_{1}, t_{2}, t_{3}$, and $t_{4}$ by 1 . Then properly color $t_{5}, v$, and $z_{5}$ in that order. Vertex $v$ will not be colored by 1, so w.l.o.g. lets assume that $v$ is properly colored by 2. If $z_{5}$ is colored by 1 , then by Lemma 17, we can color $z_{1}, z_{2}, z_{3}, z_{4}$, and $u$ by 1 and then properly color $w$, to get a coloring of $G$, a contradiction. So we can assume that $z_{5}$ is not colored by 1 . Then we properly color $z_{1}, z_{2}, z_{3}, z_{4}$ and $u$, so $w$ can have at most 3 neighbors colored by 1 , all properly. We can color $v$ by 1 to get a coloring of $G$, a contradiction.

\section{Discharging Procedure:}

We start the discharging process now. Recall that the initial charge for a vertex $v$ is $\mu(v)=2 d(v)-6$ and the initial charge for a face $f$ is $\mu(f)=d(f)-6$.

We introduce the following discharging rules:

(R1) Every 4-vertex gives 1 to each incident 3-face.

(R2) Every 5 and 6-vertex gives 2 to each incident 3-face.

(R3) every $6^{+}$-vertex gives 1 to each adjacent pendant 3 -face.

(R4) Each $d$-vertex with $7 \leq d \leq 10$ gives 3 to each incident poor $(3,3, *)$-face, 2 to each incident semi-poor 3-face, except 7 -vertices give 1 to special semi-poor 3 -face, where a special semi-poor $(3,7,7+)$-face is a semi-poor 3 -face incident to a 7 -vertex which is also incident to two poor 3 -faces and adjacent to one pendant 3 -face. Each $d$-vertex with $7 \leq d \leq 10$ gives 1 to all other incident 3 -faces.

(R5) Every $11^{+}$-vertex gives 3 to all incident 3 -faces.

Now let $v$ be a $k$-vertex. By Proposition $1, k \geq 3$.

When $k=3, v$ is not involved in the discharging process, so $\mu^{*}(v)=\mu(v)=0$.

When $k=4$, by Proposition 1, $v$ can have at most 2 incident 3 -faces. By $(\mathrm{R} 1), \mu^{*}(v) \geq$ $\mu(v)-1 \cdot 2=0$.

When $k=5$, by Proposition 1, $v$ can have at most 2 incident 3 -faces. By $(\mathrm{R} 2), \mu^{*}(v) \geq$ $\mu(v)-2 \cdot 2=0$.

When $k=6$, by Proposition 1, $v$ can have $\alpha \leq 3$ incident 3 -faces, and at most $(k-2 \alpha)$ pendant 3-faces. By (R2) and $(\mathrm{R} 3), \mu^{*}(v) \geq \mu(v)-2 \cdot \alpha-1 \cdot(k-2 \alpha)=k-6=0$.

When $k=7, v$ has an initial charge $\mu(v)=7 \cdot 2-6=8$. By Lemma 20, $v$ has at most two poor 3-faces. If $v$ has less than two incident poor 3 -faces, then by (R3) and (R4), $\mu^{*}(v) \geq \mu(v)-3 \cdot 1-1 \cdot 5=0$ since $v$ gives at most one charge per vertex excluding vertices in poor 3 -faces. So assume that $v$ has exactly 2 incident poor 3-faces. By Lemma 22, $v$ is 
adjacent to at most two pendant 3 -faces, and if it is incident to a semi-poor $\left(3,6^{-}, 7\right)$-face, then $v$ is not adjacent to a pendant 3-face. So if $v$ is not incident to a semi-poor $\left(3,7^{+}, 7\right)$ face, then by (R3) and (R4), $\mu^{*}(v) \geq \mu(v)-3 \cdot 2-2 \cdot 1=0$; If $v$ is incident to a semi-poor $\left(3,7^{+}, 7\right)$-face, then by rules (R3) and (R4), $\mu^{*}(v) \geq \mu(v)-3 \cdot 2-1 \cdot 1-1 \cdot 1=0$.

When $k=8, v$ has an initial charge $\mu(v)=8 \cdot 2-6=10$. By Lemma 20, $v$ has at most three poor 3 -faces. If $v$ has less than 3 incident poor 3 -faces, then by (R3) and (R4), $\mu^{*}(v) \geq \mu(v)-3 \cdot 2-1 \cdot 4=10-6-4=0$ since $v$ gives at most one charge per vertex excluding vertices in poor 3 -faces. So let $v$ is incident to exactly 3 poor 3 -faces. By Lemma 21, $v$ cannot be incident to a semi-poor 3-face or adjacent to two pendant 3-faces, then $\mu^{*}(v) \geq \mu(v)-3 \cdot 3-1 \cdot 1=0$.

When $k=9$, by Lemma 20, $v$ is incident to at most three poor 3 -faces. The worst case occurs when $v$ is incident 3 poor $(3,3,9)$-faces, incident one semi-poor (3,3,9)-face, and pendant one 3 -face. So by $(\mathrm{R} 3)$ and $(\mathrm{R} 4), \mu^{*}(v) \geq \mu(v)-1 \cdot 1-3 \cdot 3-2 \cdot 1=12-1-9-2=0$.

When $k=10$, by Lemma $20, v$ is incident to at most four poor $(3,3,10)$-faces. So by (R3) and (R4), $\mu^{*}(v) \geq \mu(v)-3 \cdot 4-2 \cdot 1=14-3 \cdot 4-2 \cdot 1=0$.

When $k \geq 11$, we assume that $v$ is incident to $\alpha 3$-faces, then by Proposition 1 , $\alpha \leq\lfloor k / 2\rfloor$. Thus the final charge of $v$ is $\mu^{*} \geq 2 k-6-3 \alpha-1 \cdot(k-2 \alpha)=k-\alpha-6 \geq 0$.

Now let $f$ be a $k$-face in $G$. By the conditions on $G, k=3$ or $k \geq 6$. When $k \geq 6, f$ is not involved in the discharging procedure, so $\mu *(f)=\mu(f)=k-6 \geq 0$. So in the following we only consider 3 -faces.

Case 1: $f$ is a $\left(4^{+}, 4^{+}, 4^{+}\right)$-face. By the rules, each $4^{+}$-vertex on $f$ gives at least 1 to $f$, so $\mu *(f) \geq \mu(f)+1 \cdot 3=0$.

Case 2: $f$ is a $\left(3,4^{+}, 4^{+}\right)$-face with vertices $u, v, w$ such that $d(u)=3$. If $u$ is not a poor 3-vertex, then by (R2), $f$ gains 1 from the pendant neighbor of $u$ and by the other rules, $f$ gains at least 2 from vertices on $f$, thus $\mu^{*}(f) \geq \mu(f)+1 \cdot 3=0$. If $u$ is a poor vertex (it follows that $f$ is a semi-poor 3 -face), then by Lemma $18, f$ is a $\left(3,4^{+}, 6^{+}\right)$-face. Since $v$ or $w$ is a $6^{+}$-vertex, it gives at least 2 to $f$ unless $f$ is a special semi-poor $\left(3,7,7^{+}\right)$-face, and as the other is a $4^{+}$-vertex, it gives at least 1 to $f$. Therefore, if $f$ is not a special semi-poor 3-face, then $\mu^{*}(f) \geq \mu(f)+2 \cdot 1+1 \cdot 1=0$; if $f$ is a special semi-poor $\left(3,7,8^{+}\right)$-face, then $f$ receives at least 2 from the $8^{+}$-vertex, so $\mu^{*}(v) \geq \mu(v)+2 \cdot 1+1 \cdot 1=0$. If $f$ is a special semi-poor $(3,7,7)$-face so that both $v$ and $w$ are incident to two poor 3 -faces, one semi-poor $(3,7,7)$-face and adjacent to one pendant 3 -face, then by Lemma 23 , is impossible.

Case 3: $f$ is a $\left(3,3,4^{+}\right)$-face with $4^{+}$-vertex $v$. If $d(v) \geq 11$, then by $(\mathrm{R} 5), \mu^{*}(f) \geq$ $\mu(f)+3=0$. So assume $d(v) \leq 10$. By Lemma 18 , if $4 \leq d(v) \leq 6$, then each 3-vertex has the pendant neighbor of degree 6 or higher. So by $(\mathrm{R} 1)$ and $(\mathrm{R} 3)$ (when $d(v)=4)$, $\mu^{*}(f) \geq \mu(f)+1 \cdot 3=0$, or by (R1) and (R2) (when $\left.d(v)>4\right), \mu^{*}(f)=\mu(f)+2 \cdot 1+1 \cdot 1=0$.

Let $7 \leq d(v) \leq 10$. If $f$ is poor, then by $(\mathrm{R} 4), \mu^{*}(f)=\mu(f)+3 \cdot 1=0$. If $f$ is semi-poor, then one 3 -vertex on $f$ is adjacent to a $6^{+}$-vertex and thus by (R3) $f$ gains 1 from it, together the 2 that $f$ gains from $v$ by $(\mathrm{R} 4)$, we have $\mu^{*}(f)=\mu(f)+2 \cdot 1+1 \cdot 1=0$. If $f$ is non-poor, then both 3 -vertices on $f$ are adjacent to the pendant neighbors of degrees more than 5 , thus by $(\mathrm{R} 3)$ and $(\mathrm{R} 4), \mu^{*}(f)=\mu(f)+1 \cdot 2+1 \cdot 1=0$.

Case 4: $f$ is a $(3,3,3)$-face. By Lemma 18, each 3-vertex will have the pendant neighbor of degree 6 or higher, so by (R3), $\mu^{*}(f)=\mu(f)+1 \cdot 3=0$.

Since for all $x \in V \cup F, \mu^{*}(x) \geq 0, \sum_{v \in V} \mu^{*}(v)+\sum_{f \in F} \mu^{*}(f) \geq 0$, a contradiction. This completes the proof of Theorem 1.2. 


\section{ACKNOWLEDGEMENT}

The research is supported in part by NSA grant H98230-12-1-0226 and NSF CSUMS grant. The authors thank Bernard Lidicky for some preliminary discussion.

\section{REFERENCES}

[1] O.V. Borodin, Colorings of planar graphs: a survey. Disc. Math., to appear.

[2] O. V. Borodin, A. N. Glebov, A. R. Raspaud, and M. R. Salavatipour. Planar graphs without cycles of length from 4 to 7 are 3-colorable. J. of Comb. Theory, Ser. B, 93 (2005), 303-311.

[3] G. Chang, F. Havet, M. Montassier, and A. Raspaud, Steinberg's Conjecture and near colorings, preprint.

[4] N. Eaton and T. Hull. Defective list colorings of planar graphs. Bull. Inst. Combin. Appl., 25 (1999), $78-87$.

[5] H. Grötzsch, Ein dreifarbensatz f ur dreikreisfreienetze auf der kugel. Math.-Nat.Reihe, 8 (1959), 109120.

[6] R. Škrekovski. List improper coloring of planar graphs. Comb. Prob. Comp., 8 (1999), 293299.

[7] R. Steinberg, The state of the three color problem. Quo Vadis, Graph Theory?, Ann. Discrete Math. 55 (1993), 211-248.

[8] B. Xu, On $(3,1)^{*}$-coloring of planar graphs, SIAM J. Disc. Math., 23 (2008), 205-220.

Department of Mathematics, College of William and Mary, Williamsburg, VA 23185.

E-mail address: oshill@email.wm.edu, gyu@wm.edu 